12.20-12.30 h Cutaneous T-Cell Lymphoma: Value and Prognostic Significance of a PCR-Detectable T-Cell Clone B. Niklaus, J. Benhattar, N. Kunzle, F. Burri, W. Kempf, R.G. Panizzon, Lausanne, Zürich

12.30-12.40 h Topische Behandlung chronischer Wunden mit dem Anti-TNF- $\alpha$-Antikörper Infliximab M. Streit, L.R. Braathen, Bern

12.40-12.50 h Une déficience d'expression de CD40L est une cause de la faible production d'IL- 12 et TNF- $\alpha$ dans le syndrome de Sézary: correction par une forme soluble hexamérique de CD40L

L.E. French, B. Huard, M. Wysocka, R. Shane,

E. Contassot, J.-F. Arrighi, V. Piguet, S. Calderara, A.H. Rook, Genève, Epalinges, Philadelphia

12.50-13.30 h Stehlunch, Besuch der Poster- und Industrieausstellung Lunch, visite des posters et de l'exposition de l'industrie

13.30-14.00 h Klinische Demonstrationen/Démonstrations cliniques Dermatologische Abteilung, Kantonsspital Aarau

14.00-14.30 h Klinische Demonstrationen/Démonstrations cliniques Dermatologische Klinik, Universitätsspital Zürich

14.30-15.00 h Klinische Demonstrationen/Démonstrations cliniques Dermatologische Klinik, Universitätsspital Basel

15.00-16.30 h Berufspolitisches Seminar/Séminaire de politique professionnelle

Die Berufspolitik der Dermatologen in Frankreich und Deutschland/La politique professionnelle des dermatologues en France et en Allemagne

Für Frankreich/pour la France:

Dr. Georges Reuter, Secrétaire général de la section et du board de dermatologie et de vénéréologie de l'UEMS

Für Deutschland/pour l'Allemagne:

Dr. Martin Schläger, Präsident der Deutschen

Dermatologischen Akademie (Sonderbeauftragter des Präsidiums des BVDD für Qulitätssicherung,

Moderatoren und Leitlinien)

Schluss der Jahresversammlung/Clôture de la Réunion annuelle

\section{Allgemeine Informationen Informations générales}

\section{Tagungsorte/Lieux des congrès}

(siehe Situationsplan Seite 22/Veuillez vous reporter au plan de situation page 22)

\section{Universitätsspital Basel}

27.10.2004: Vorstandssitzung/ Gartensaal des Holsteinerhofes Séance du comité Hebelstrasse 32 (L)

28.10.2004: Vorstandssitzung Gartensaal des Holsteinerhofes Séance du comité Hebelstrasse 32 (L)

28.10.2004: Workshops der Arbeitsgruppen SGDV (A, D, M) Ateliers des groupes de travail de la SSDV Schweizer Pflegefachgruppe Dermatologie (D) Satelliten-Symposium Essex Chemie (D) Satelliten-Symposium Innéov Suisse SA (D)

\section{Kongresszentrum Messe Basel}

29./30.10.2004: Jahresversammlung SGDV/Réunion annuelle de la SSDV

\section{Wissenschaftliche Organisation/Organisation scientifique}

Prof. T. Rufli (Kongresspräsident/Président du congrès)

Frau N. Sulzer E-Mail trufli@uhbs.ch Tel. 0612654084 Fax 0612654885

Prof. A. Bircher E-Mail nsulzer@uhbs.ch

Prof. S. Büchner

Prof. P. Itin

Koordination Workshops der Arbeitsgruppen SGDV/ Coordination des Ateliers des groupes de travail de la SSDV Dr. J. Izakovic, Tel. 06126528 30, E-Mail jizakovic@uhbs.ch

\section{Administratives Sekretariat/Secrétariat administratif}

Convention Team Lucerne AG, Oberseeburg 10, 6006 Luzern, Tel. 04137118 60, Fax 04137118 61, E-Mail ctlag@bluewin.ch

\section{Während des Kongresses/Pendant le congrès}

Tel. 06169205 12, Fax 0616819429

\section{Kongresssprachen/Langues du congrès}

Deutsch, Französisch/Allemand, français

(keine Simultanübersetzung/pas de traduction simultanée)

Kongressgebühr/Frais d'inscription

Mitglieder SGDV, Assistenten, Studenten

Membres SSDV, assistants, étudiants

Nicht-Mitglieder SGDV (ganzer Kongress)

Non-membres (tout le congrès)

Nicht-Mitglieder SGDV (1 Tag)

Non-membres (seulement 1 jour)

Galadiner/Dîner de gala

Stehlunch/Lunch (30.10.2004)

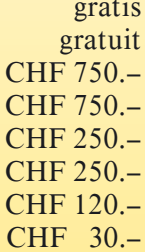

\section{Credits/Crédits}

Es können geltend gemacht werden/vous pouvez faire valoir:

Workshop 28.10.2004

JV 1. Tag/RA 1er jour 4 Credits/Crédits

JV 2. Tag/RA 2e jour 


\section{Informations générales Allgemeine Informationen}

Freie Mitteilungen/Communications libres

$8 \mathrm{~min}+2 \mathrm{~min}$ Diskussion $/ 8 \mathrm{~min}+2 \mathrm{~min}$ discussion

Diaprojektoren (Einzel- und Doppelprojektion) mit Karussell stehen zur Verfügung. Dia-Abgabe: Freitag und Samstag ab $8.00 \mathrm{~h}$,

Raum Hongkong; Abgabe der ppt-Files: Freitag 8.00-9.00 h und 12.30-13.30 h in der Projektionskabine, Saal Montreal, oder Samstag $8.00-9.00 \mathrm{~h}$

Projecteurs (projection simple et double) plus des carrousels sont à la disposition des orateurs. Remise des diapositives: vendredi et samedi dès 8.00 h, salle Hongkong; remise des files ppt: vendredi 8.00-9.00 h et $12.30-13.30 \mathrm{~h}$ dans la cabine de projection, salle Montréal, ou samedi 8.00-9.00 h

\section{Anmeldung/Hotelreservation/Inscription/Réservation} d'hôtels

Für Hotelreservationen wenden Sie sich bitte an:

Pour la réservation d'hôtel, veuillez vous adresser à:

Basel Tourismus, Incoming Services

Tel. +416126868 58, Fax +41612686870

E-Mailincoming@baseltourismus.ch

\section{Öffentliche Verkehrsmittel/Transport public}

Universitätsspital:

Vom Hauptbahnhof: Tram Nr. 8 (Haltestelle: Schifflände),

+ Tram Nr. 11 (Haltestelle: Kantonsspital) oder Bus Nr. 30 (Haltestelle: Frauenspital)

De la gare: Tram No. 8 (arrêt: Schifflände) + Tram No. 11

(arrêt: Kantonsspital) ou Bus No. 30 (arrêt: Frauenspital)

Kongresszentrum Messe Basel:

Vom Hauptbahnhof: Tram Nr. 2 (Haltestelle: Messeplatz)

De la gare: Tram No. 2 (arrêt: Messeplatz)

\section{Parking}

Universitätsspital: Parkhaus/parking City

Kongresszentrum Messe Basel: Parkhaus/parking Messe Basel

(Ausfahrtstickets können am Kongressdesk gekauft werden/Des tickets de sortie peuvent être achetés au bureau du congrès)

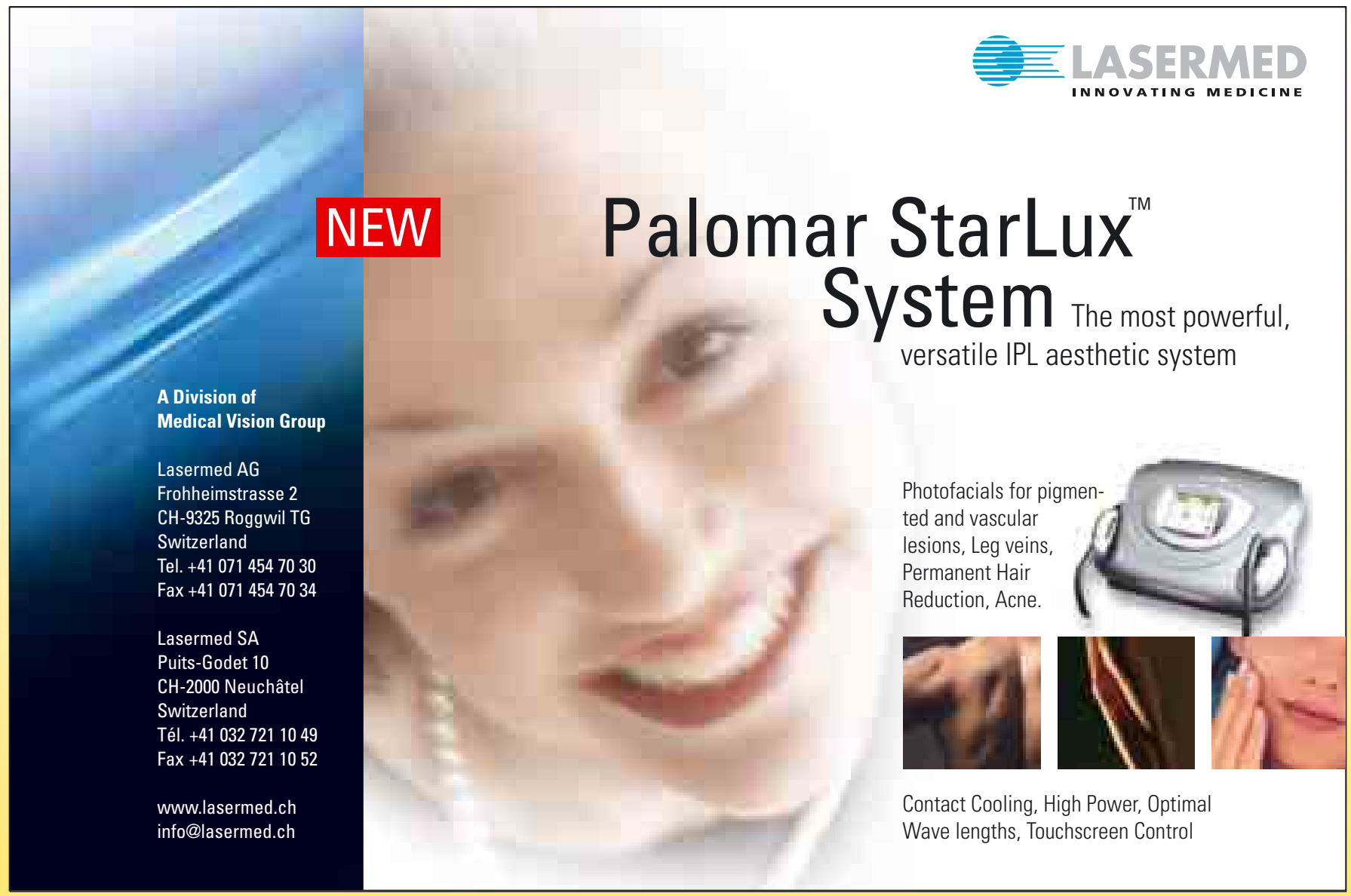




\section{Sponsorenliste/Liste des sponsor}

Speziell möchten wir folgenden Firmen für ihre grosszügige Unterstützung danken/Nous remercions spécialement les firmes suivantes de leur généreux support

Platin-Sponsor/Médaille de platine

Novartis Pharma Schweiz AG Bern

Gold-Sponsor/Médaille d'or

Essex Chemie AG Luzern

Galderma SA Cham

Mepha Pharma AG Aesch

Silber-Sponsor/Médaille d'argent

GlaxoSmithKline AG

Roche Pharma (Schweiz) AG

Münchenbuchsee

Reinach

Egerkingen

Spirig Pharma AG

Therwil

Bronze-Sponsor/Médaille de bronze Laboratoire Vichy

Neuenhof

\section{Liste der Aussteller/Liste des exposants}

Wir danken folgenden Firmen herzlich für ihr Interesse und ihre Beteiligung (bis 16. August 2004)

Nous remercions vivement pour leur participation et leur intérêt les firmes suivantes (jusqu'au 16 août 2004)

\begin{tabular}{ll}
$\begin{array}{l}\text { Aha! Schweizerisches Zentrum } \\
\text { für Allergie, Haut und Asthma }\end{array}$ & Bern \\
\hline Allergomed & Therwil \\
\hline ASS-Trade GmbH & Rotkreuz \\
\hline Beiersdorf AG (Geschäftsbereich Eucerin) & Volketswil \\
\hline Biogen-Dompé AG & Zug \\
\hline Bluemedic Sàrl & Paudex \\
\hline Boots Healthcare (Switzerland) AG & Zug \\
\hline Calista & Wallisellen \\
\hline DebriTec & Horgen \\
\hline Drossapharm AG & Arlesheim \\
\hline Enzym-Labor Dr. H. Weber AG & St. Gallen \\
\hline Essex Chemie AG & Luzern \\
\hline Esthetic-Med & Zürich \\
\hline Fujisawa AG & Wallisellen \\
\hline Galderma AG & Cham \\
\hline Ganzoni \& Cie Sigvaris & St. Gallen \\
\hline Gebro Pharma AG & Liestal \\
\hline GlaxoSmithKline AG & Münchenbuchsee \\
\hline Globopharm AG & Küsnacht \\
\hline GMP & Renens \\
\hline IBSA & Pambio-Noranco \\
\hline ICN Pharmaceuticals Switzerland AG & Birsfelden \\
\hline Inneov Suisse SA & Neuenhof \\
\hline Innothera CH & St-Blaise \\
\hline Janssen-Cilag AG & Baar \\
\hline Johnson \& Johnson & Spreitenbach \\
\hline & \\
\hline
\end{tabular}

\begin{tabular}{|c|c|}
\hline S. Karger AG & Basel \\
\hline KCI Medical GmbH & Geroldswil \\
\hline La Roche-Posay & Neuenhof \\
\hline Laboratoires Vichy & Neuenhof \\
\hline Lasermed AG & Roggwil \\
\hline Leo Pharmaceutical Products & Zürich \\
\hline Louis Widmer SA & Schlieren \\
\hline 3M (Schweiz) AG & Rüschlikon \\
\hline Medic Service AG & Tagelswangen \\
\hline Medika AG & Aesch \\
\hline Medilas AG & Geroldswil \\
\hline Medinova AG & Zürich \\
\hline A. Menarini AG & Zürich \\
\hline Mepha Pharma AG & Aesch \\
\hline Merz Pharma & Allschwil \\
\hline Mölnlycke Health Care AG & Dietikon \\
\hline NMS Bio Medical SA & Praroman \\
\hline Novartis Pharma Schweiz AG & Bern \\
\hline Omnimedica AG & Schlieren \\
\hline Panpharma SA & Fribourg \\
\hline Permamed AG & Therwil \\
\hline Pierre Fabre (Suisse) SA & Crissier \\
\hline Procter \& Gamble & $\begin{array}{l}\text { Schwalbach } \\
\text { (Deutschland) }\end{array}$ \\
\hline Qualicare AG (Stiefel Vertretung) & Basel \\
\hline Roche Pharma (Schweiz) AG & Reinach \\
\hline Sanatrend AG & Neerach \\
\hline Schweiz. Psoriasis- und Vitiligo-Gesellschaft & Zürich \\
\hline Serono Pharma Schweiz & Zug \\
\hline Shan Shui & Lachen \\
\hline Spirig Pharma AG & Egerkingen \\
\hline Trimedal AG & Brüttisellen \\
\hline UCB-Pharma AG & Zürich \\
\hline Viollier AG & Basel \\
\hline Waldmann Lichttechnik GmbH & Küttigen \\
\hline Wyeth & Zug \\
\hline
\end{tabular}




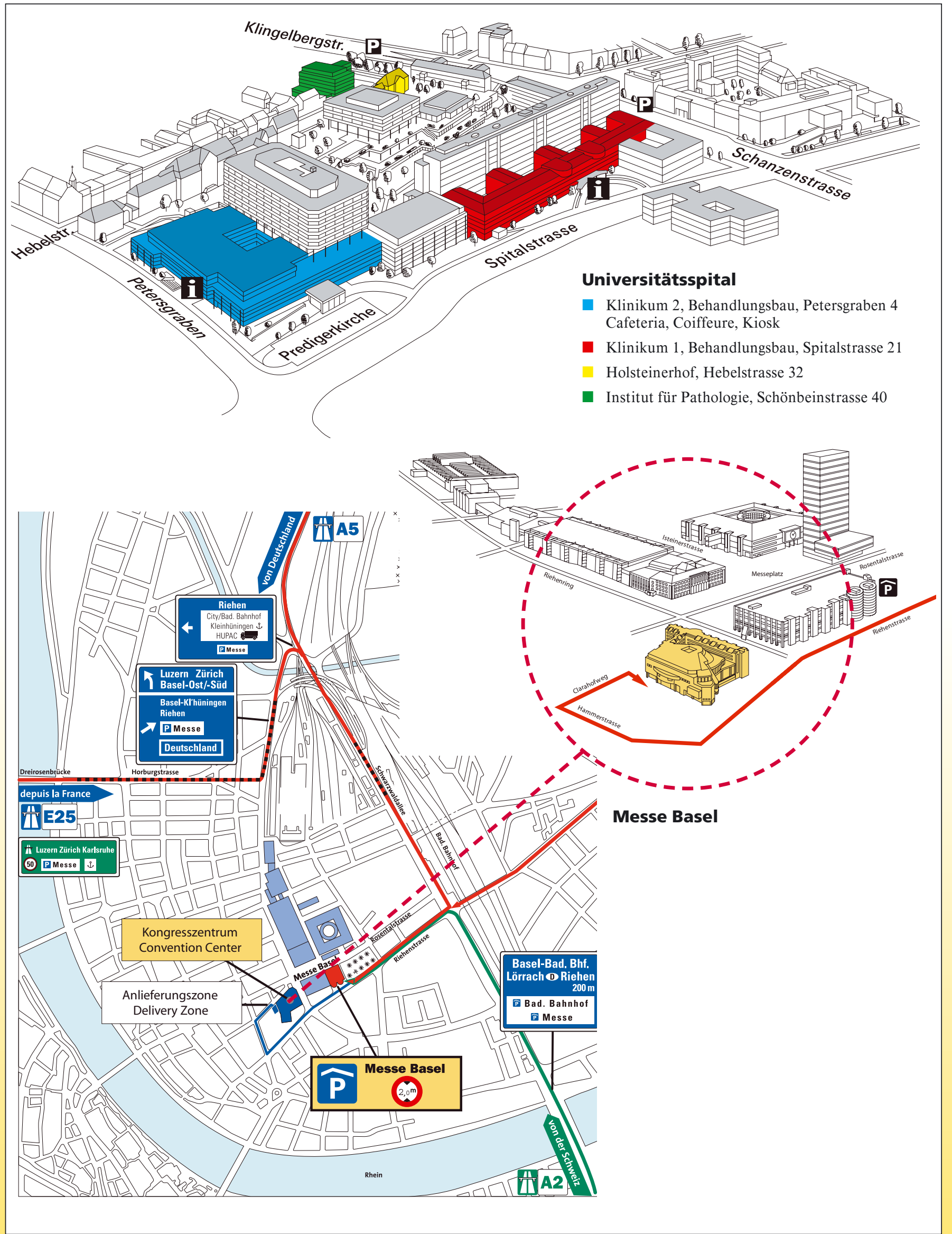

